\title{
Application of Magnetic Coupling Resonant Wireless Power Supply in a Torque Online Telemetering System of a Rolling Mill
}

\author{
Jinliang Jia $(\mathbb{D}$ and Xiaoqiang Yan $(\mathbb{D})$ \\ School of Mechanical Engineering, University of Science and Technology Beijing, Beijing 100083, China \\ Correspondence should be addressed to Xiaoqiang Yan; yanxq@ustb.edu.cn
}

Received 25 February 2020; Accepted 29 April 2020; Published 14 May 2020

Academic Editor: François Vallée

Copyright (c) 2020 Jinliang Jia and Xiaoqiang Yan. This is an open access article distributed under the Creative Commons Attribution License, which permits unrestricted use, distribution, and reproduction in any medium, provided the original work is properly cited.

\begin{abstract}
The torque of the main drive system is one of the most important force and energy parameters of the rolling mill, and the strain type torque online telemetry system is a practical method for measuring torque parameters. The strain gauges and transmitters on the rotating shaft are driven by a high-frequency induction power system. Their installation, debugging, and maintenance are cumbersome, and their very low power transmission efficiency (PTE) has become a challenging problem for online telemetry systems. In this paper, a magnetic coupling resonant wireless power supply method is utilized to replace the highfrequency induction power supply. Through theoretical and experimental research, it is concluded that the magnetically coupled resonant wireless power supply method can realize long-distance power supply, overcoming many shortcomings of the high-frequency induction power supply system. In the laboratory, a maximum PTE of $83.7 \%$ is obtained for a transmission distance of $50 \mathrm{~mm}$. Under the influence of environmental factors common for the transmission shaft of a rolling mill, the PTE decreases by $34 \%$, but normal operation of the system can be achieved by adjusting the compensation capacitance. The proposed system provides a guarantee for a long-term stable power supply on the measured axis of a rolling mill torque online telemetry system.
\end{abstract}

\section{Introduction}

The main drive torque of a rolling mill is one of the most important force and energy parameters in the rolling process. It not only reflects the load of the main drive system, but also reflects the dynamic property of the torque. Therefore, online real-time monitoring of the torque signal can aid in optimizing the rolling schedule, maximizing the efficiency of the rolling mill, accurately determining faults, and avoiding production accidents, making the rolling mill safe, efficient, and reliable.

Presently, the domestic online rolling mill torque telemetry system mainly depends on imports from the United States. The system consists of the wireless transmission of torque signals and a high-frequency induction power supply. The high import price, cumbersome installation, and poor after-sales service have a great impact on the application of the system. The domestic wireless signal transmission technology is mature, but the field application stability of high-frequency induction power supply technology is poor.

After many years of research, University of Science and Technology Beijing has made great progress on a domestic online remote measuring system for rolling mill torque [1]. The proposed magnetically coupled resonant wireless power supply system replaces the high-frequency induction power supply system [2] (Figure 1). It eliminates the need for strict adjustment of the installation gap between the static power supply ring and the rotating ring $(3-7 \mathrm{~mm}$ is generally required), enables long-distance misalignment installation, eases disassembly of the inner ring on-site (without first disassembling the outer ring), and solves many problems related to field installation, debugging, installation, maintenance, and other shortcomings.

In recent years, magnetically coupled resonant radio power transmission technology has become a challenging global research hotspot. The system design, topology 


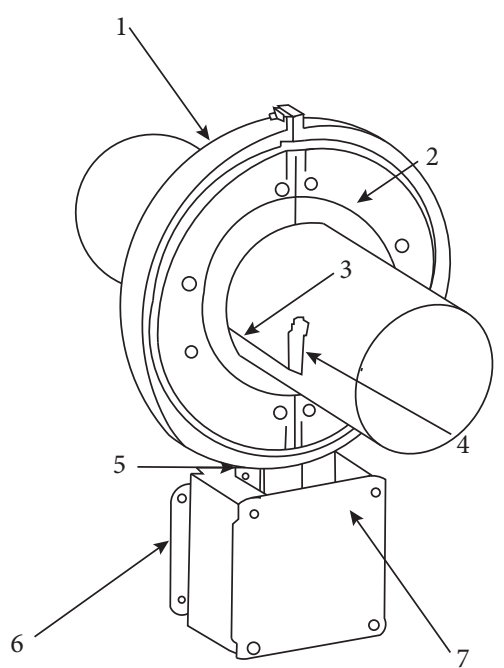

Figure 1: Component assembly diagram of the original torque telemetry system. 1-static power ring, 2-rotating ring, 3-strain gauge conductor, 4 -welded strain gauge, 5 -system state indicator lamp, 6-flange, 7-main control unit.

structure, model establishment, and simulation of magnetic coupling resonance wireless power transfer (MCRWPT) technology have been deeply studied, and great progress has been made. In 2007, the US MIT research team took the lead in proposing MCRWPT technology and successfully lit $60 \mathrm{~W}$ light bulbs with $40 \%$ PTE at a $2 \mathrm{~m}$ distance [3]. In 2008, the Intel Seattle Laboratory Research Group achieved $60 \mathrm{~W}$ power transmission within $1 \mathrm{~m}$ with $70 \%$ PTE [4]. In 2009, Tokyo University of Japan established a functional relationship between the resonant frequency and the relative position of the resonant coil and conducted an experimental study [5]. In 2011, a Stanford University scientific research team theoretically analyzed $10 \mathrm{~kW}$ power transmission within $1.98 \mathrm{~m}$ [6]. In 2013, Florida International University found that the PTE of magnetic resonance coupling in different media can be optimized [7]. In 2018, Yonsei University proposed that an asymmetric coil can improve the efficiency and the degree of freedom in terms of position. At transmission distances of $50 \mathrm{~mm}$ and $300 \mathrm{~mm}$, they realized transmission efficiencies of $96 \%$ and $39 \%$, respectively [8]. South Korea Advanced Institute of Science and Technology designed a T-type ferrite wireless power transmission system and operated the system in an experiment with the transmission of $205 \mathrm{~W}$ power and a total efficiency of $71 \%$ [9].

The difference between the principle of induction coupling and magnetic coupling system was examined and analyzed at South China University of Technology, whose work provided a theoretical basis for further optimizing the two system models [10]. Research at Hebei University of Technology showed that impedance matching has a large influence on PTE and output power, and their work proposed an optimization method for PTE and output power [11]. By establishing a T-type equivalent circuit model, Tianjin University of Technology obtained the dynamic transmission curve and constraint function of a transmission system and found the functional relationship of maximizing PTE [12]. From the analysis of a seriesparallel equivalent circuit model, Southeast University concluded that output power and PTE are closely related to frequency, and the optimal operating frequency was obtained through simulation and experimental analysis [13]. Through the study of a dual relay wireless power transmission system at Southeast University, it was concluded that output power and PTE are the highest when the optimal turn value exists in the receiving coil [14]. Through several parameter circuit models of a transmission system, the conditions of maximum load power under different coupling conditions were obtained [15]. Chongqing University used equivalent circuit theory to model and analyze a system, provided the method to calculate the optimal frequency of PTE and optimal frequency of transmission power, proposed concepts of optimal resonant frequency and an efficiency synchronization factor, and obtained the conditions of synchronization between PTE and maximum power [16]. A parameter dynamic adjustment method based on a chaos optimization algorithm proposed by Hunan University improved the efficiency of a noncoaxial system coil, which has significance for the analysis of radio power transmission systems [17]. Research at Hebei University found that the single resonance structure of multisource coils can reduce the load voltage change caused by the magnetic field distribution by utilizing the coupling between loads [18].

Research institutions for MCRWPT technology transfer efficiency and studies of power transfer theory are ample. However, there is a lack of experience in the development and application of engineering practice and equipment. Research and problem-solving in this area is a challenge. In the work presented here, MCRWPT technology is studied in the torque telemetry system of a rolling mill to evaluate an engineering application and gain practical experience.

\section{Theoretical Research}

2.1. System Composition. MCRWPT technology uses a space electromagnetic field as a medium, uses two or more electromagnetic systems with the same resonant frequency and high quality factor, and realizes wireless power transmission through a magnetic coupling resonant method. A highfrequency induction power supply is based on the principle of electromagnetic induction using a high-frequency power supply to generate a magnetic field with air as a magnetic medium to make an inner and outer loop coil induction power supply. The installation distance between the inner and outer loop coils must be within several millimeters to supply normal induction power. MCRWPT technology, which utilizes the magnetic coupling resonant technology, can achieve high-efficiency power transmission with a larger dislocation range of the inner and outer rings, and it can facilitate installation of the rolling mill torque online telemetry system.

The MCRWPT system consists of four parts: a power supply module, an electromagnetic transmitting module, an electromagnetic receiving module, and a load module, as 
shown in Figure 2. The power supply module is composed of a rectifier and a high-frequency inverter, which provides suitable resonant frequency voltage input for the electromagnetic transmitting module. The electromagnetic transmitting module converts a high-frequency input voltage into a high-frequency magnetic field, and strong magnetic coupling occurs between the space electromagnetic field and the electromagnetic receiving module. The electromagnetic receiving module converts magnetic energy into electric energy and outputs it to the load module through constant voltage rectification. The electromagnetic transmitting and receiving modules are key parts of the power supply system. Functionality of the transmitting and receiving modules in the resonant state ensures the power transmission. Therefore, the transmitting and receiving modules are composed of high quality factor resonant coils with identical structures and parameters and a resonant compensation capacitor. In the torque telemetry system, the transmitter and strain gauge on the measured axis are used as load modules to consume energy. To ensure optimal PTE of the system, the impedance at the load end and the internal resistance at the power supply end are always matched.

Cylindrical spiral coils and plane spiral coils, as shown in Figure 3, are primarily used [19]. The magnetic field intensity at the edge of the helical coil is greater than that the center. The magnetic induction intensity of the planar disc coil decreases from inside to outside, and the magnetic induction intensity is the largest at the center of the coil. The two coil structures have their own characteristics and can be selected according to application requirements.

2.2. Theoretical Analysis. To optimize the power transmission efficiency, it is usually necessary to compensate for the capacitance of the resonant coil to reach the resonant state. The compensation circuit is divided into four forms: SeriesSeries (SS), Series-Parallel (SP), Parallel-Parallel (PP), and Parallel-Series (PS), as shown in Figure 4. To achieve the resonant state of the system, the imaginary part of the total resistance of the system after capacitance compensation is zero. The compensation capacitance of the SP, PP, and PS structures is related to the load resistance, and only the compensation capacitance of the receiving and transmitting terminals of SS structures is independent of the load. Because the load resistance of the online torque telemetry system varies during its operation, an SS structure is chosen for the capacitance compensation circuit in this paper.

Presently, there are two main MCRWPT research methods: coupled mode theory and equivalent circuit theory. Each research method has its own benefits. Coupled mode theory is a mathematical method that describes a complex coupled system as an energy transmission process of an independent single system from the perspective of energy, simplifying and clarifying the analysis and calculation. Equivalent circuit theory establishes an equivalent circuit model based on the basic principle of a circuit and the equivalent calculation method, which is intuitive and easy to understand. Depending on the specific parameters of the circuit, the voltage and current changes in the circuit are analyzed to calculate the PTE and power of the system electric energy. In this paper, equivalent circuit theory is used to study the process of power transmission. Its equivalent circuit model is shown in Figure 5.

In Figure 5, $U_{S}$ represents high-frequency power supply excitation, $R_{S}$ represents internal resistance of power supply, $R_{1}$ represents equivalent resistance of transmitter, $C_{1}$ represents compensation capacitance of transmitter, $L_{1}$ represents transmitting coil, $L_{2}$ represents receiving coil, $R_{2}$ represents equivalent resistance of receiver, $C_{2}$ represents compensation capacitance of receiver, $R_{L}$ represents load resistance, $M$ represents mutual inductance between transmitting coil and receiving coil, $i_{1}$ represents loop current of transmitter, and $i_{2}$ represents loop current of receiver.

According to Kirchhoff's law, the equations of the circuit in Figure 5 are listed in (1) and (2):

$$
\begin{gathered}
R_{S} i_{1}+R_{1} i_{1}+j \omega L_{1} i_{1}+\frac{i_{1}}{j \omega C_{1}}-j \omega M i_{2}=U_{S}, \\
-j \omega M i_{1}+j \omega L_{2} i_{2}+R_{2} i_{2}+R_{L} i_{2}+\frac{i_{2}}{j \omega C_{2}}=0 .
\end{gathered}
$$

The simultaneous solutions to (1) and (2) give the current of the transmitting and receiving coils, respectively:

$$
\begin{aligned}
& i_{1}=\frac{U_{S}}{R_{S}+R_{1}+j \omega L_{1}+\left(1 / j \omega C_{1}\right)-\left(j \omega M / R_{2}+R_{L}+j \omega L_{2}+\left(1 / j \omega C_{2}\right)\right)}, \\
& i_{2}=\frac{j \omega M U_{S}}{\left(R_{2}+R_{L}+j \omega L_{2}+\left(1 / j \omega C_{2}\right)\right)\left(R_{S}+R_{1}+j \omega L_{1}+\left(1 / j \omega C_{1}\right)\right)-(j \omega M)^{2}} .
\end{aligned}
$$

$$
\begin{aligned}
& i_{1}=\frac{U_{S} R}{R^{2}-(j \omega M)^{2}}, \\
& i_{2}=\frac{j \omega M U_{S}}{R^{2}-(j \omega M)^{2}} .
\end{aligned}
$$

and parameters of the transmitting and receiving coils are identical, and the impedance of the transmitting and receiving ends matches. Therefore, if $C_{1}=C_{2}=C, L_{1}=L_{2}=L$, $\omega=2 \pi f=(1 / \sqrt{L C})$, and $R_{1}+R_{S}=R_{2}+R_{L}=R$, the system PTE of the system is maximized. Thus, formulas (3) and (4) can be simplified as follows:
From this, it can be concluded that the output and input power of the system are, respectively, 


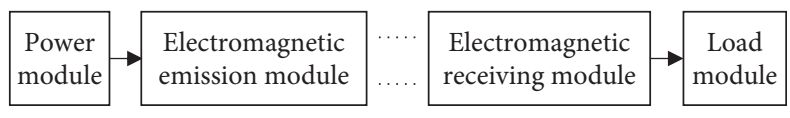

FIGURE 2: Structure diagram of MCRWPT system.

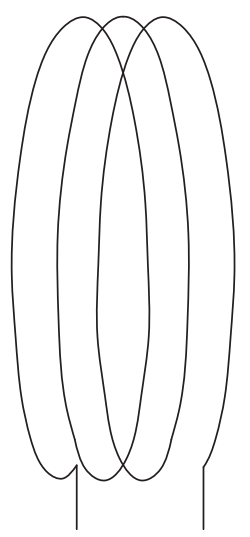

(a)

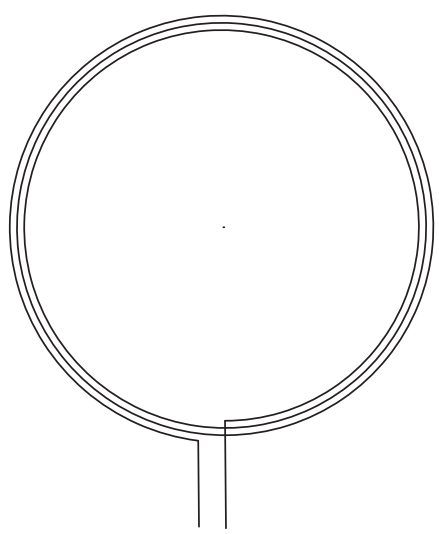

(b)

FIGURE 3: Structure diagram of a MCRWPT system. (a) Cylindrical spiral coil. (b) Planar spiral coil.

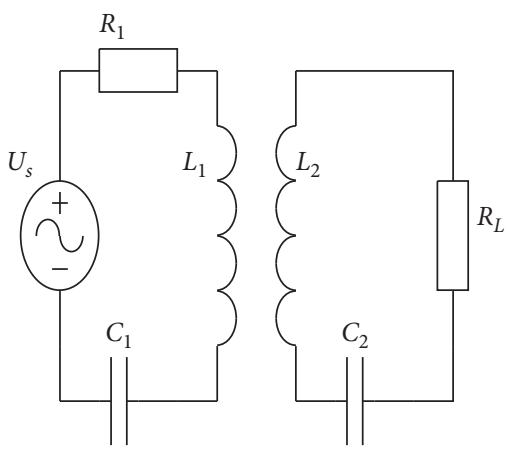

(a)

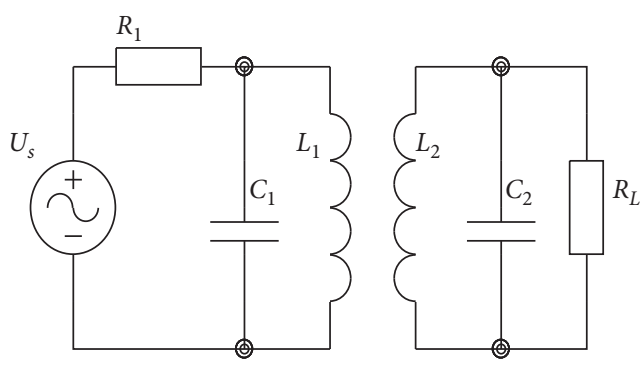

(c)

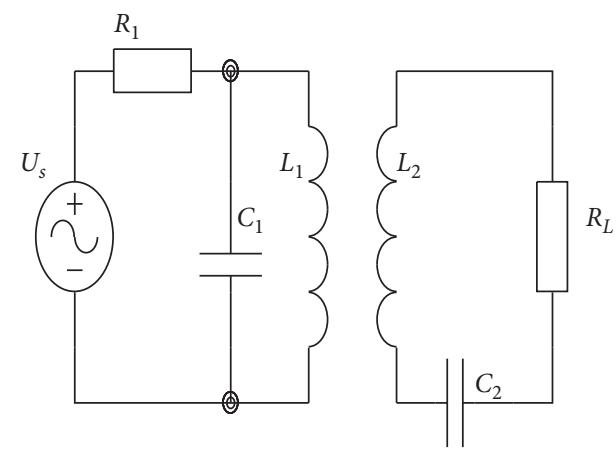

(b)

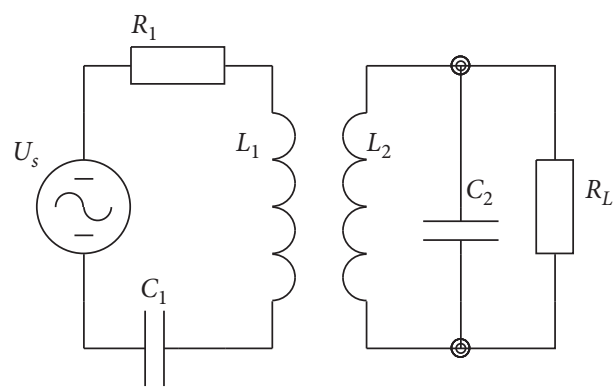

(d)

FIgURE 4: Capacitance compensation circuit. (a) SS structure. (b) SP structure. (c) PP structure. (d) PS structure.

$$
\begin{aligned}
P_{\text {in }} & =U_{S} i_{1}+i_{1}^{2} R_{s}=\frac{U_{S}^{2} R\left(R^{2}+\omega^{2} M^{2}\right)+U_{S}^{2} R^{2} R_{s}}{\left(R^{2}+\omega^{2} M^{2}\right)^{2}}, \\
P_{\text {out }} & =i_{2}^{2} R_{L}=\frac{\omega^{2} M^{2} U_{S}^{2} R_{L}}{\left(R^{2}+\omega^{2} M^{2}\right)^{2}} .
\end{aligned}
$$

From formulas (6) and (7), the PTE of the system can be calculated as follows:

$$
\eta=\frac{P_{\text {out }}}{P_{\text {in }}}=\frac{\omega^{2} M^{2} R_{L}}{\left(R^{3}+R^{2} R_{s}+\omega^{2} M^{2}\right)} .
$$




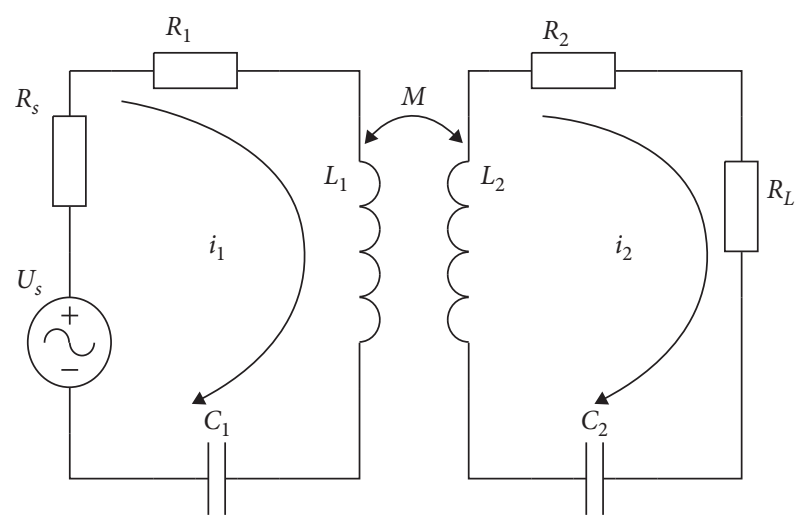

Figure 5: Equivalent circuit of MCRWPT.

It can be seen from formula (8) that the PTE of the power supply system is related to the resonant frequency of the system, the mutual inductance value $M$ between coils, and the resistance value of the system. The resistance value of the system includes the equivalent resistance value of the transmitting and receiving coils, the internal resistance of the power supply, and the load resistance value. The PTE of the system can be obtained by substituting the angular frequency $\omega=2 \pi f$ into formula (8):

$$
\eta=\frac{4 \pi^{2} f^{2} M^{2} R_{L}}{\left(R^{3}+R^{2} R_{s}+4 \pi^{2} f^{2} M^{2}\right)} .
$$

From the above analysis, it can be seen that changing the resonant frequency $f$, mutual inductance $M$, and resistance $R$ of the system can improve the PTE of the power supply system.

The equivalent resistance of the system depends on the quality factor of the coil used in the system, which is defined as

$$
Q=\frac{\omega L}{R}
$$

In the formula, $\omega$ is the working angle frequency, $L$ is the inductance of the coil, and $R$ is the total loss resistance of the coil. It is composed of DC resistance and dielectric loss of high-frequency resistance. The resonant frequency of the system is related to the parameters of the system hardware and the power supply frequency. The mutual inductance of coils depends on the material, radius, turns, and the distance between the two coils.

The calculation method for coil mutual inductance $M$ is given in [20] and formula (11).

$$
M=\frac{\pi \mu_{0} \sqrt{n_{1} n_{2}}\left(r_{1} r_{2}\right)^{2}}{2 D^{3}} .
$$

In the formula, $\mu_{0}=4 \pi \times 10^{-7} \mathrm{H} \cdot \mathrm{m}^{-1}$ is the vacuum permeability; $n_{1}, n_{2}$ and $r_{1}, r_{2}$ are the turns and radius of the transmitting coil and the receiving coil, respectively; and $D$ is the distance between the transmitting coil and the receiving coil. When the diameter of the measured axis is determined, the radius of the resonant coil is fixed, and the mutual inductance value is mainly determined by the number of turns of the coil and the distance between the coils. Parameters for the example of the measured shaft of a rolling mill are shown in Table 1.
TABLE 1: Modeling parameter.

\begin{tabular}{lc}
\hline Parameter name & Numerical value \\
\hline Drive shaft diameter $(\mathrm{mm})$ & $200 \sim 900$ \\
Coil diameter $(\mathrm{mm})$ & $200 \sim 900$ \\
Coil turns & 5 \\
Power supply amplitude $(\mathrm{V})$ & 5 \\
Power frequency $(\mathrm{MHz})$ & $1 \sim 10$ \\
Resistance value of telemetering device $(\Omega)$ & 325 \\
\hline
\end{tabular}

The self-resistance of the coil is [21]

$$
R=\sqrt{\frac{\omega \mu_{0}}{2 \sigma}} \frac{l}{2 \pi a}
$$

In the formula, $\sigma=5.7 \times 107 \mathrm{~s} \cdot \mathrm{m}^{-1}$ is the conductivity of the coil copper wire, $l$ is the length of the coil, and $a$ is the diameter of the coil.

Evaluation with the Origin data analysis software shows that the PTE of the system varies as the resonant frequency of the system varies from 1 to $10 \mathrm{MHz}$, the coil spacing varies from 50 to $600 \mathrm{~mm}$, and the coil diameter is set to 200, 400, 700 , and $900 \mathrm{~mm}$. The analyzed cases are shown in Figure 6.

Figure 6(a) shows that when the coil diameter is $200 \mathrm{~mm}$ and the distance between transmitting and receiving coils is more than $100 \mathrm{~mm}$, the PTE of the system is close to zero. When the distance between transmitting and receiving coils is less than $100 \mathrm{~mm}$, the PTE of the system observably changes, with a smaller distance between coils corresponding to a higher system PTE. Increasing the resonant frequency of the system improves the PTE, providing significant benefits. Figure 6(d) shows that when the coil diameter is $900 \mathrm{~mm}$, the PTE of the system observably changes with a change in resonant frequency and coil spacing. When the frequency increases and the coil spacing decreases, the PTE of the system increases continuously, and when the coil spacing is less than $200 \mathrm{~mm}$, the PTE of the system exceeds $50 \%$. Through comparison, it can be seen that when the coil diameter is larger, a change in coil spacing and frequency has a greater impact on the PTE, and a higher PTE can be maintained. When the coil diameter is smaller, the overall PTE of the system is low, and only by reducing the coil spacing can the PTE of the system be significantly improved.

The results of the theoretical study show that the PTE of a power supply system varies with the resonant frequency, the coil spacing, and the coil diameter. When applied to a rolling mill, depending on the diameter of the axis installed in a telemetry system and the field environment, the power supply system can achieve higher PTE by choosing appropriate power frequency, matching capacitance, and coil spacing for a given coil diameter.

\section{Experimental Results and Analysis}

3.1. Experimental Design and Analysis. Based on the theoretical research results, coils with diameters of $700 \mathrm{~mm}$ and $400 \mathrm{~mm}$ are experimentally studied as shown in Figure 7.

The power supply frequency is $5 \mathrm{MHz}$, and the coil diameter is $700 \mathrm{~mm}$ and $400 \mathrm{~mm}$, respectively. When the coil diameter is $700 \mathrm{~mm}$ and the coil spacing is $50 \mathrm{~mm}$, the 


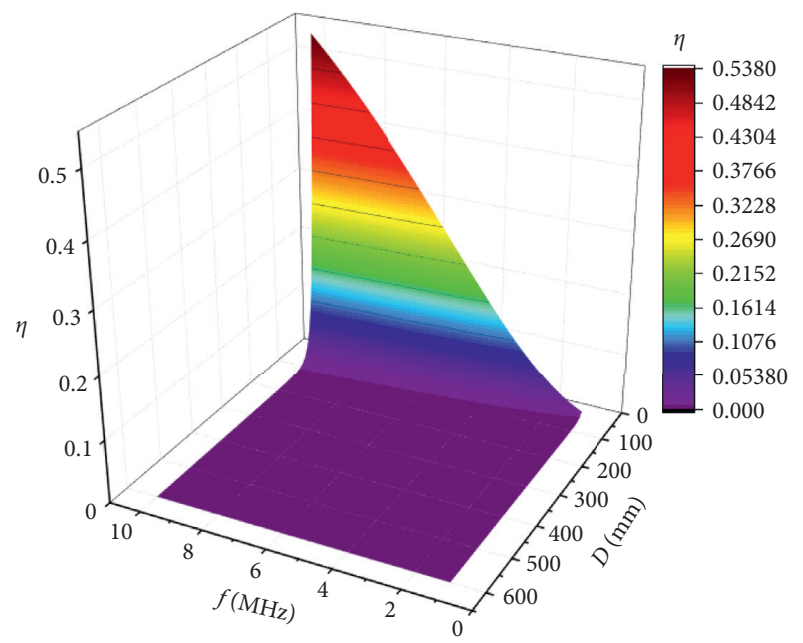

(a)

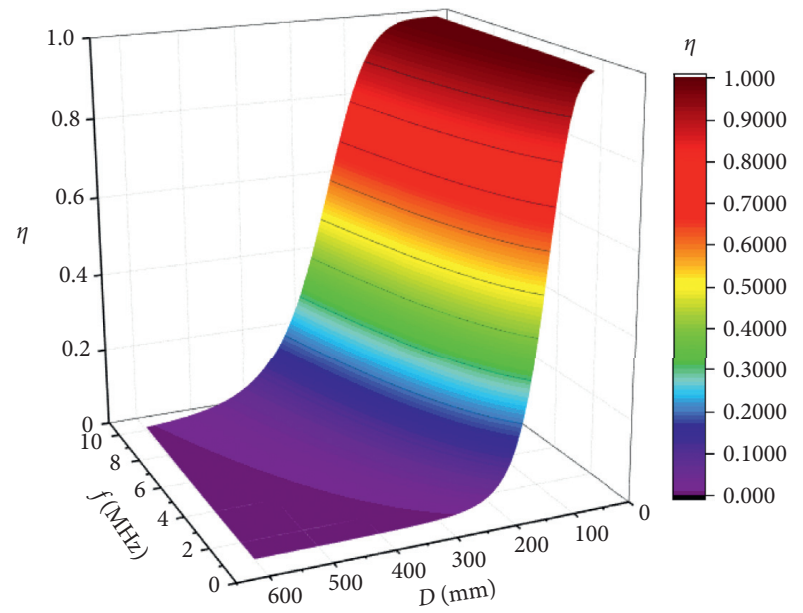

(c)

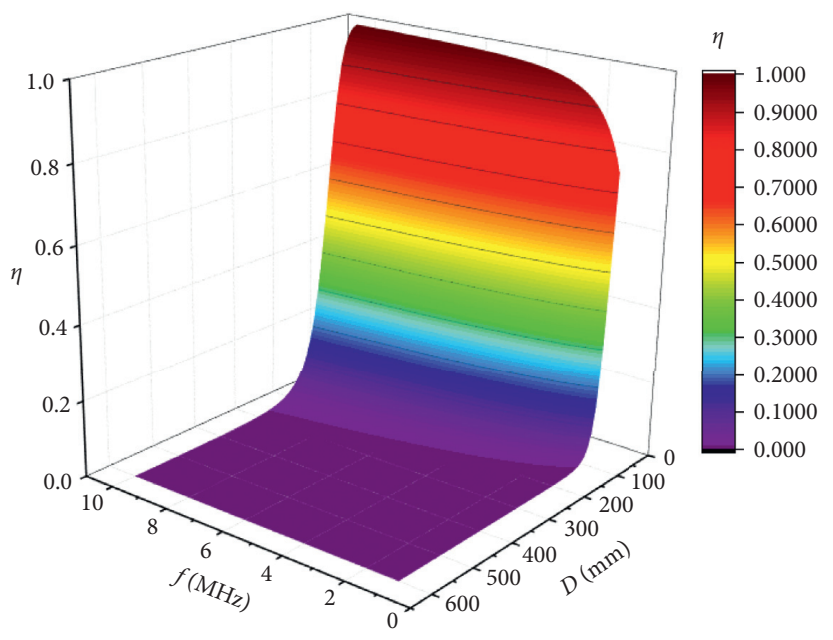

(b)

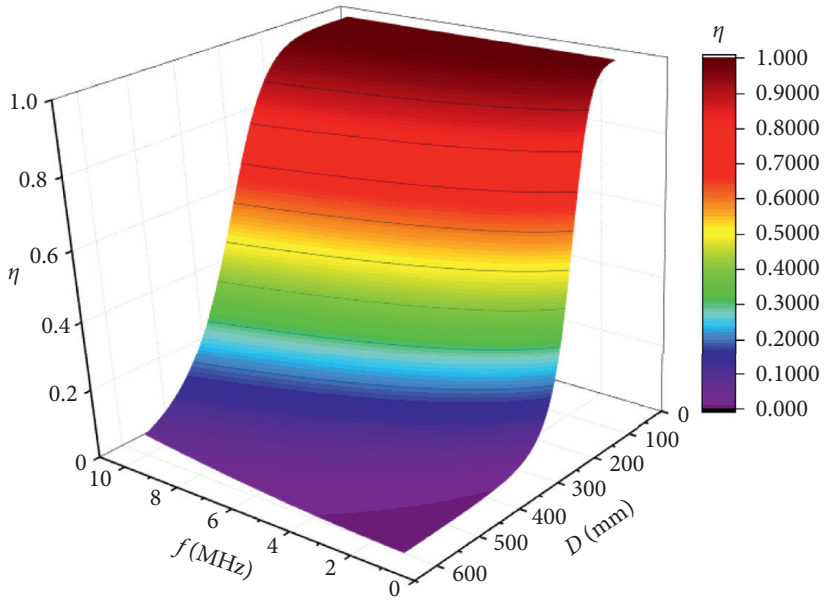

(d)

Figure 6: Variation of $\eta$ with f and D. (a) Coil diameter of $200 \mathrm{~mm}$. (b) Coil diameter of $400 \mathrm{~mm}$. (c) Coil diameter of $700 \mathrm{~mm}$. (d) Coil diameter of $900 \mathrm{~mm}$.

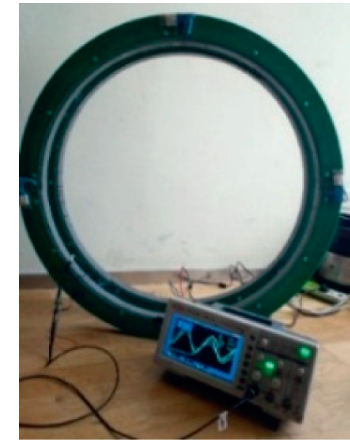

(a)

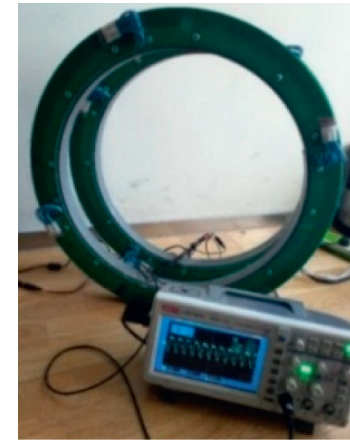

(b)

Figure 7: Experimental device setup photos. (a) Coil diameter $700 \mathrm{~mm}$. (b) Coil diameter $400 \mathrm{~mm}$.

waveforms of the primary coil supply voltage and the secondary coil induction voltage are obtained as shown in Figure 8.

The relationship between PTE and coil spacing is obtained by changing coil spacing. A comparison of PTE in terms of the experimentally and theoretically calculated efficiency of the system is shown in Figure 9.

From Figure 9, it can be seen that the PTE varies with coil spacing when the resonant frequency is fixed. For the $400 \mathrm{~mm}$ coil diameter, the PTE of the system changes 


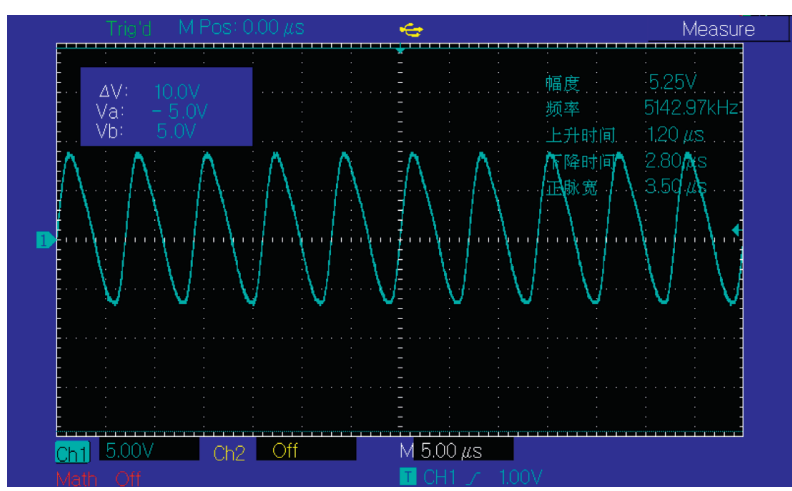

(a)

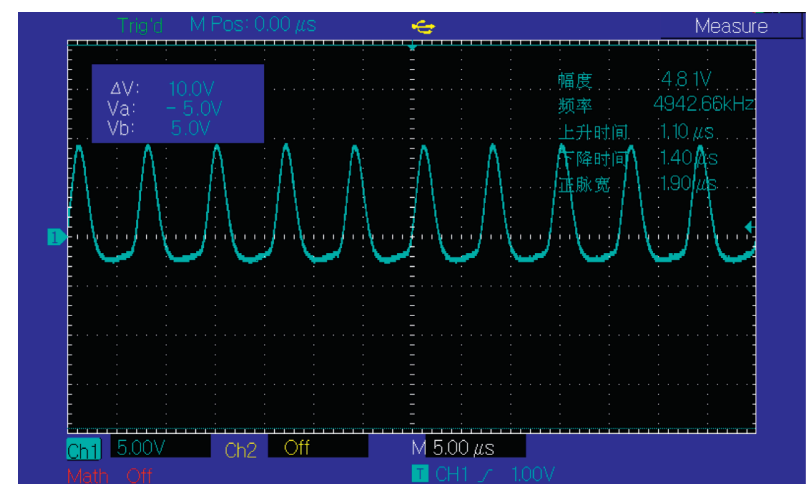

(b)

FIGURE 8: Experimental waveform diagrams. (a) Voltage waveform of primary coil supply. (b) Induced voltage waveform of secondary coil.

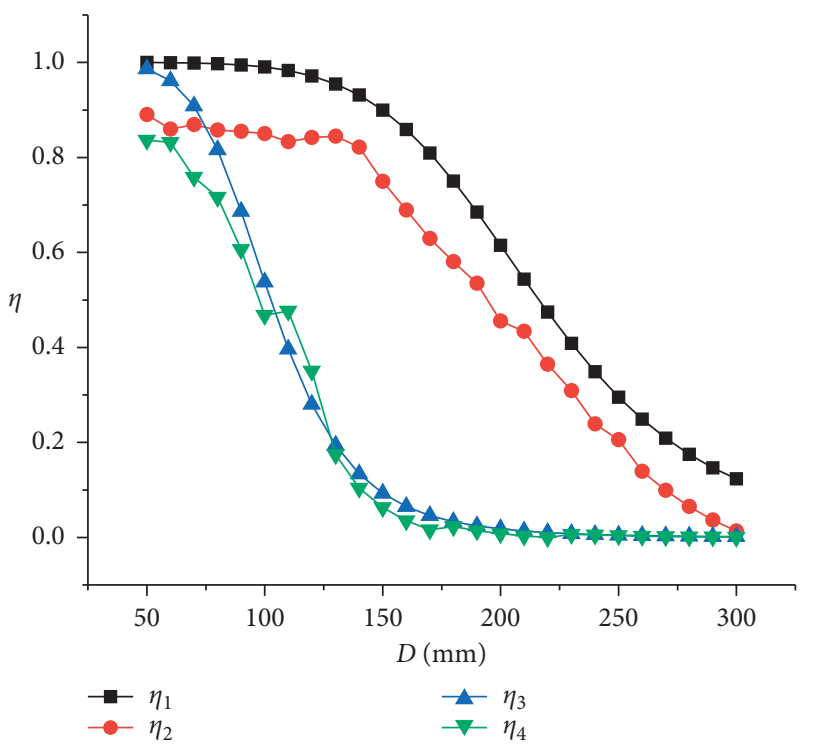

FIGURE 9: Simulation and experimental efficiency comparison chart.

rapidly, and a high efficiency of power transmission can be maintained only when the coil spacing is less than $100 \mathrm{~mm}$. When the coil diameter is $700 \mathrm{~mm}$, the overall PTE of the system is greatly improved. The PTE of the system can still exceed $50 \%$ with coil spacing up to $250 \mathrm{~mm}$. The experimental PTE trend is largely consistent with the results of theoretical research.

The developed rolling mill torque telemetry system based on magnetic coupling resonant wireless power supply has been installed and applied on the output axles of main drive motors of several continuous rolling mills, as shown in Figure 10(a). A limitation of the original inductive power supply mode is that the transmission distance cannot exceed approximately $7 \mathrm{~mm}$ to achieve stable power transmission. A magnetically coupled wireless power supply removes that limitation and can supply power efficiently and steadily over a large distance (generally approximately $200 \mathrm{~mm}$ ), which significantly eases system installation and maintenance.
During the manufacturing and debugging of the equipment, the induction coil centers of the primary and secondary sides of the telemetry system pass through the axis, while the drive shaft of the rolling mill passes through the coil centers after being installed on-site. The presence of the drive shaft of a rolling mill changes the inductance of the coil and the mutual inductance between the coils, which makes the system unable to work in the resonant state and results in a decrease in PTE and power.

The field test results for the $400 \mathrm{~mm}$ diameter drive shaft are shown in Figure 10, and the test data results for the $400 \mathrm{~mm}$ shaft are shown in Table 2.

Table 2 shows that the primary side inductance change rate is $12.9 \%$, the secondary side inductance change rate is $12.8 \%$, and the PTE is reduced by $34 \%$. The change of inductance under the influence of a transmission shaft can change the resonant state of the system. By adjusting the capacitance of the primary side resonant circuit, the system 


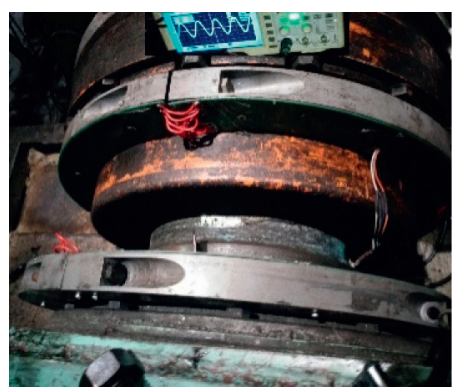

(a)

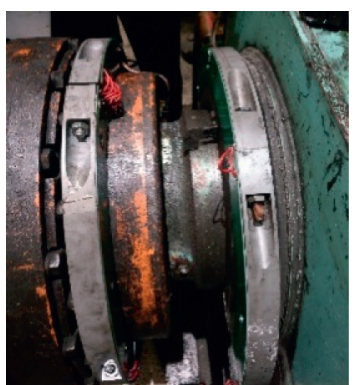

(b)

Figure 10: Field test setup photos.

TABLE 2: Field test data results.

\begin{tabular}{|c|c|c|c|c|c|c|c|}
\hline \multicolumn{3}{|c|}{ Change of primary inductance } & \multicolumn{3}{|c|}{ Secondary inductance change } & \multirow{2}{*}{$\Delta U_{2}$} & \multirow{2}{*}{$\triangle \eta(\%$} \\
\hline$\underline{L_{1}}$ & $L_{1}^{\prime}$ & $\triangle L_{1}$ & $L_{2}$ & $L_{2}^{\prime}$ & $\Delta L_{2}$ & & \\
\hline 9.78 & 8.52 & 1.26 & 9.67 & 8.43 & 1.24 & 2.1 & -34 \\
\hline
\end{tabular}

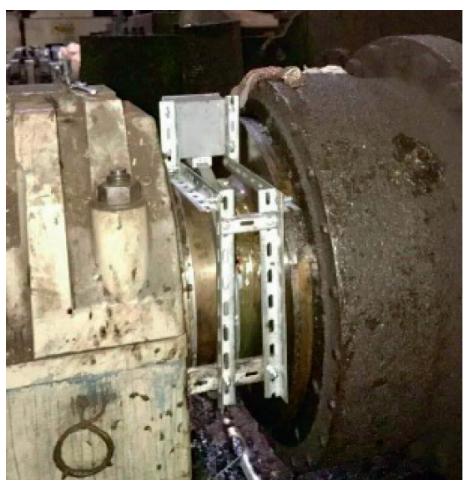

(a)
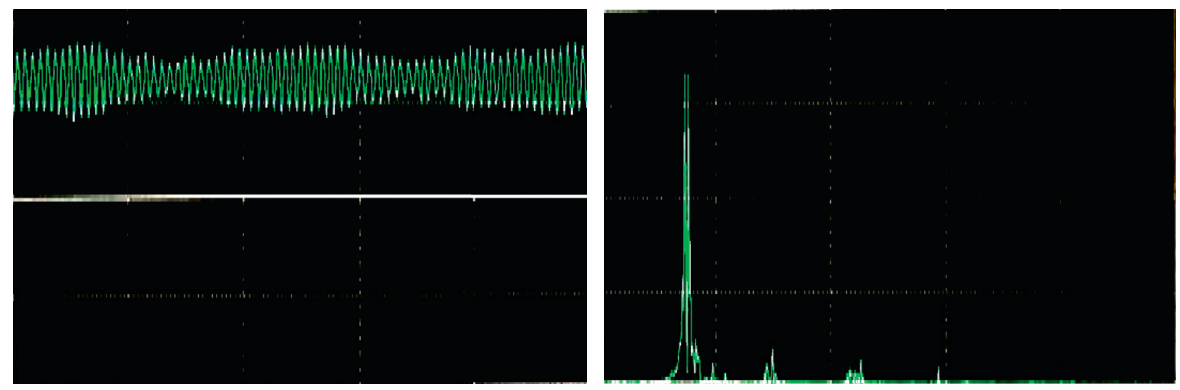

(b)

Figure 11: Installed system and torque signal time domain analysis diagrams. (a) Photo of an on-site torque telemetry device. (b) Torque signal waveform and spectrum plots.

can maintain a high PTE, thus ensuring that the telemetry equipment can maintain its normal working state.

The system has been running steadily for more than one year in several continuous rolling mills as shown in Figure 11(a). The real-time waveform of the collected torque signal is shown in Figure 11(b).

\section{Conclusions}

In this paper, the power supply of a rotating ring in an online torque telemetry system is studied. The developed magnetic coupling resonant wireless power supply system replaces the high-frequency induction power supply system. The relationship between mutual inductance, coil spacing, and PTE was obtained through mathematical derivation. Origin data analysis software was used to simulate the variation trend of system PTE for different coil diameters and transmission spacing. By designing an experimental platform to test the power transfer efficiency when the coil diameter is $700 \mathrm{~mm}$ and $400 \mathrm{~mm}$ when the pitch is $50 \mathrm{~mm}$, the results show that the simulation results are basically consistent with the experimental results. In the field application, the PTE of the radio power transmission system was reduced by $34 \%$ due to the influence of the transmission shaft of the rolling mill. An adjustment of the primary compensation capacitance can allow the system to meet the normal power demand and ensure normal operation. The influence of the transmission shaft on the efficiency of power transmission in a telemetry system is the next key research direction.

\section{Data Availability}

The datasets supporting the conclusions of this article are included within the article.

\section{Conflicts of Interest}

The authors declare no conflicts of interest. 


\section{Acknowledgments}

This work was financially supported by 12th Five-Year National Science and Technology Support Plan, Precision Strip Steel Product Quality Optimization and Key Equipment Research and Development (Grant no. 2015BAF30B00), and Fundamental Research Funds for the Central Universities (Grant no. FRF-AT-19-001).

\section{References}

[1] X. Q. Yan and X. B. Cui, "Torque telemetering system of main drive system for rolling mill based on nRF9E5," Journal of Microcomputer Information, vol. 29, no. 1-9, pp. 107-108, 2007.

[2] X. Q. Yan, H. Zhang, and S. L. Yang, "Torque monitor system of main drive system for rolling mill," Journal of Metallurgical Equipment, vol. 12, no. 6, pp. 63-66, 2001.

[3] X. M. Fan, X. Y. Mo, and X. Zhang, "Research status and application of wireless power transfer via coupled magnetic resonances," Journal of Transactions of China Electrotechnical Society, vol. 28, no. 12, pp. 75-82, 2013.

[4] Z. M. Zhao, Y. M. Zhang, and K. N. Chen, "New progress of magnetically-coupled resonant wireless power transfer technology," Journal of Proceedings of the CSEE, vol. 33, no. 3, pp. 1-13, 2013.

[5] C. Chen, "Research on electromagnetic problems and optimization design of magnetic resonant wireless power transfer system," Doctoral Dissertation, Southeast University, Nanjing, China, 2016.

[6] F. X. Yang, "Research on key technologies of wireless power transfer networks based on inductively coupled power transfer," Doctoral Dissertation, Southeast University, Nanjing, China, 2012.

[7] O. Jonah, "Optomization of wireless power transfer via magnetic resonance in different media,"Doctoral Dissertation, Florida International University, Miami, FL, USA, 2013.

[8] T.-H. Kim, G.-H. Yun, W. Y. Lee, and J.-G. Yook, “Asymmetric coil structures for highly efficient wireless power transfer systems," IEEE Transactions on Microwave Theory and Techniques, vol. 66, no. 7, pp. 3443-3451, 2018.

[9] Y. Shin, J. Park, and J. Kim, Wireless Power Transfer System for Unmanned Vehicle Using T-Shape Ferrite Structure, Wiley, Hoboken, NJ, USA, 2018.

[10] X. J. Shu and B. Zhang, "Energy model and characteristic analysis for inductively coupled power transfer system," Journal of Automation of Electric Power System, vol. 41, no. 2, pp. 28-32, 2017.

[11] Y. Li, Q. X. Yang, and H. Y. Chen, "Analysis of factors influencing power and efficiency in wireless power transfer system," Journal of Advanced Technology of Electrical Engineering and Energy, vol. 31, no. 3, pp. 31-34, 2012.

[12] X. Zhang, Q. X. Yang, and H. Y. Chen, "Modeling and design and experimental verification of contactless power transmission systems via electromagnetic resonant coupling," Journal of Proceedings of the CSEE, vol. 32, no. 21, pp. 153-158, 2012.

[13] X. L. Huang, J. J. Ji, and L. L. Tan, "Study on series-parallel model of wireless power transfer via magnetic resonance coupling," Journal of Transactions of China Electrotechnical Society, vol. 28, no. 3, pp. 171-176, 2013.

[14] W. Wang, X. L. Huang, and Y. L. Zhou, "Modeling and PTE analysis of wireless power transmission system with dual relays," Journal of Transactions of China Electrotechnical Society, vol. 29, no. 9, pp. 1-6, 2014.

[15] R. W. Porto, V. J. Brusamarello, I. Müller, F. L. Cabrera Riaño, and F. Rangel De Sousa, "Wireless power transfer for contactless instrumentation and measurement," IEEE Instrumentation \& Measurement Magazine, vol. 20, no. 4, pp. 49-54, 2017.

[16] Z. D. Tang, F. Yang, and Y. Y. Xu, "Research on power efficiency synchronization of wireless power transfer magnetic resonant coupling," Journal of Transactions of China Electrotechnical Society, vol. 32, pp. 190-197, 2017.

[17] Z. Q. Li, S. D. Huang, and X. F. Yuan, "A method of preventing frequency splitting in magnetic resonant wireless power transfer system," Journal of Transactions of China Electrotechnical Society, vol. 32, no. 8, pp. 152-159, 2017.

[18] Z. Yan, T. F. Wang, and X. C. Zhang, "Optimization of the source coil of magnetic coupling resonant wireless power transmission system with class E power amplifier," Journal of Transactions of China Electrotechnical Society, vol. 32, no. 10, pp. 162-167, 2017.

[19] L. L. Tan, X. L. Huang, and J. F. Zhao, "Optimization design for disc resonators of a wireless power transmission system," Journal of Transactions of China Electrotechnical Society, vol. 28, no. 8, pp. 1-6, 2013.

[20] Y. Sun, L. Zhang, and Z. H. Wang, "Constant voltage output of wireless power transfer system based on AC envelope modulation," Journal of Automation of Electric Power System, vol. 41, no. 2, pp. 33-37, 2017.

[21] S. Assawaworrarit, X. Yu, and S. Fan, "Robust wireless power transfer using a nonlinear parity-time-symmetric circuit," Nature, vol. 546, no. 7658, pp. 387-390, 2017. 